\title{
The use of cable-screw construct as an effective method for direct pars repair
}

\begin{abstract}
Background: The aim of this study is to assess the use of cable-wire construct together with bone grafting for direct repair of pars defects in spondylolysis.

Patient and methods: Thirty patients with bilateral pars defect in complaining of low back pain were included in this prospective study. A cable-screw construct was used for pars repair after failure of conservative measures. Patients were assessed radiologically for union and clinically by Oswestry disability index (ODI) and VAS for back pain. Demographic data and any complications were noted.

Results: The mean follow up period was 22 months. The mean preoperative ODI and VAS were 67.77 and 8.2 respectively. This improved to 11.07 and 1.33 at final follow up. The improvements were statistically significant. At the final follow up, radiological healing could be achieved bilaterally in all patients $(86.6 \%)$ but four. Three of them had initial defects $>3 \mathrm{~mm}$ and 1 from $2-3 \mathrm{~mm}$. For the healed cases, the mean time to union was 4.7 months.
\end{abstract}

Conclusion: The cable-screw construct appears to be a rigid, low profile construct with high success rates in treating pars defects both radiologically and clinically.
Volume 9 Issue 5 - 2017

\author{
Abdel-Mohsen Arafa,' Hany El Zahlawy, ${ }^{2}$ \\ Abdelrady Mahmoud Abdelrady ${ }^{2}$ \\ 'Professor of Orthopedics, Faculty of Medicine, Ain Shams \\ University, Egypt \\ ${ }^{2}$ Lecturer of Orthopedics, Faculty of Medicine, Ain Shams \\ University, Egypt
}

Correspondence: Hany El Zahlawy, Department of Orthopedics, Faculty of Medicine, Ain Shams University, 18 Rabaa Housing Project, El Nasr city, Cairo, Egypt, Tel 201000000000, Fax 20224509378, Email hzahlawy@hotmail.com

Received: December 08, 2017 | Published: December 27, 2017

\section{Introduction}

Spondylolysis is a condition seen in approximately $6-8 \%$ of the population. ${ }^{1}$ It involves a defect in the pars interarticularis precipitated by repeated low grade trauma resulting in a stress fracture. The defect is occupied by fibrocartilagenous tissue resulting in nonunion. ${ }^{2}$ The prevalance is higher among athletes regularly performig hyperextension and rotation activities to the lumbar spine, reaching up to $63 \%{ }^{3}$

The condition may be asymptomatic, found incidentally on plain radiographs, or causing disabling back pain aggravated by sports. ${ }^{3}$ In some cases the patients may present with radicular symptoms. The mainstay of treatment is non-surgical, involving rest in athletes, analgesics, bracing and physiotherapy.,5

However, surgery may be required in those not responding to conservative measures. Fusion of the involved vertebra with the adjoining caudal one is often performed as standard procedure in symptomatic spndylolysis, either posterolateral or interbody. 6,7

Direct pars repair has been recommended by some, particularly in younger patients with minimal vertebral slippage and healthy discs. It reduces risk of adjacent segment disease seen after fusion procedures and maintains spinal mobility. Fusion may have devastating consequence on the long term as most patients are adolescents to young adults with athletic ambitions. ${ }^{8}$

Various techniques have been reported such as Buck technique using translaminar interfragmentary screws, Scott wiring technique, hook screw constructs and pedicle screw rod constructs. ${ }^{9-13}$ The most important step in these operations is autogenous bone grafting of the defects after thorough debridement of the intervening tissues.

Some of those have been technically difficult to perform or biomechanically inadequate to handle the forces across the pars interarticularis resulting in high failure rates. ${ }^{14}$ In this study, we present a modified technique for direct pars repair using screws across the pedicles and sublaminar cables, together with the results of the procedure clinically and radiologically.

\section{Materials and methods}

Thirty consecutive patients were included in this prospective study. The patients complained of low back pain with no radicular symptoms. Diagnostic images (plain radiographs and CT) revealed bilateral pars defect in all patients. They were considered for surgical pars repair after failure of at least 6 months of conservative treatment (medications, rest from strenuous activities, bracing and physiotherapy) with persistence of disabling pain. Only patients with spondylolisthesis less than $3 \mathrm{~mm}$ and MRI showing no degenerated discs below the slipped vertebra or type 2 Modic changes were considered as surgical candidates. Patients with unilateral pars defects were excluded from this study. As a preoperative diagnostic test, the pars defects were injected with saline for pain provocation, followed by injection of $1 \%$ saline for pain relief. Those with positive tests were included in the study.

The surgical technique involved placing $4.5 \mathrm{~mm}$ fully threaded cortical screws with washers into the pedicles of the affected vertebra for most of their lengths. A $1 \mathrm{~mm}$ titanium double ended atlas cable with ball and socket ends was passed underneath the laminae from caudal to cranial then cut (Figure 1). Each end was passed around the head of the contralateral screw and wrapped around the spinous process cranially then passed through the ball and socket end. The cables were simultaneously tensioned and crimped. The soft tissues in the pars defect was thoroughly debrided then burred to square it off for fitting a tricortical iliac bone graft which was compressed across the defect while tensioning the cables (Figure 2).

The wire is passed underneath the lamina from caudal to cranial then cut. (B) Each end is passed around the head of the contralateral screw and wrapped around the spinous process cranially then passed through the ball and socket end. The cables were simultaneously tensioned and crimped. Note the bone graft filling the pars defect. 


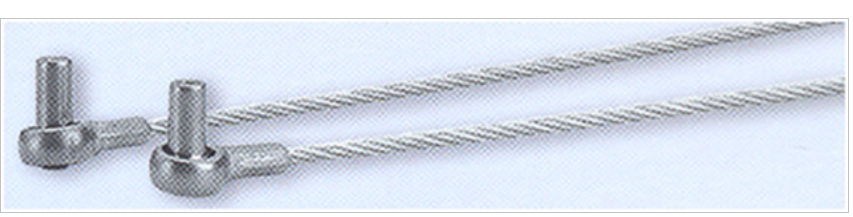

Figure I A I mm titanium double ended atlas cable with ball and socket ends was used.

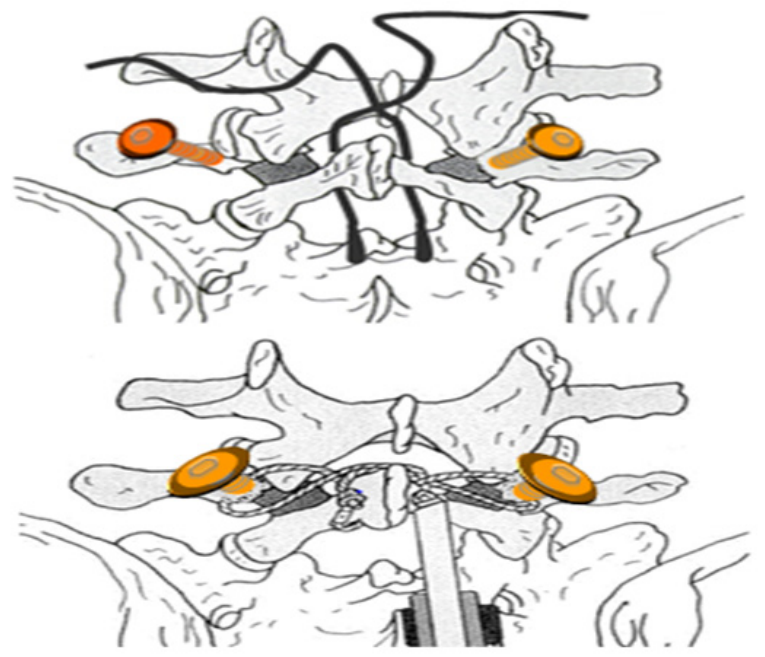

Figure 2 The construct. (A) $4.5 \mathrm{~mm}$ fully threaded cortical screws with washers are placed into the pedicles.

The demographic data of the patients were recorded. Other documented parameters included the duration of symptoms, the levels affected, size of the defect as seen on CT and the presence of spondylolisthesis $(<3 \mathrm{~mm})$. Mean operative time, blood loss and any complications related to the procedure were also noted.

Clinically, the patients were followed up using Oswestry disability index (ODI) and visual analogue scale (VAS) for back pain. The patients were followed up for at least 1 year and the values were recorded and compared preoperatively, 3 months postoperatively and at final follow up. Patients were followed up radiologically using plain radiographs and CT images. They were performed monthly in the first 6 months postoperatively then every 3 months to assess union at the defect site and any implant failure. Statistical analysis was performed using paired t tests where $p$ values $\leq 0.05$ was considered statistically significant.

\section{Results}

Twenty six males and 4 females were included in this study. Their mean age was 24.3 years (range 16-38). Only three patients were athletes in this study. The mean duration of symptoms was 14.3 months. 20 patients had the pars defects at L5 whereas 8 at L4. 2 patients had pars defects in double levels; both L4 and L5. The defect size was $1-2 \mathrm{~mm}$ in 16 levels, $2-3 \mathrm{~mm}$ in $10,>3 \mathrm{~mm}$ in 6 . Spondylolisthesis $(<3 \mathrm{~mm})$ was found in 5 cases, all at L5/S1.

The mean operative time was 110 minutes with mean blood loss of $126 \mathrm{ml}$. All patients were allowed to ambulate on the second day of surgery without a corset. Postoperatively two patients suffered from superficial wound infection which resolved with antibiotics. One suffered from anterior thigh numbness from positioning for 3 months.

The mean follow up period was 22 months (18-26 months). The mean preoperative ODI was 67.77 . This improved to 25.73 at 3 months after surgery and 11.07 at final follow up. The improvements were statistically significant. As regard VAS for back pain, the preoperative mean of 8.2 improved to 4.1 and 1.33 at 3 months and final follow up respectively. The changes were also statistically significant (Table 1).

Table I Clinical parameters before and after surgery at final follow up

\begin{tabular}{llll}
\hline & Preoperative & At final follow up & P-value \\
\hline ODI & 67.77 & $\mathrm{II} .07$ & 0.03 \\
VAS for Back Pain & 8.2 & 1.33 & 0.023 \\
\hline
\end{tabular}

No implant failure was detected in the follow up radiographs. At the final follow up, radiological healing could be achieved bilaterally in all patients $(86.6 \%)$ but four. Three of them had initial defects $>3 \mathrm{~mm}$ and one from $2-3 \mathrm{~mm}$, all at L5. For the healed cases, the mean time to union was 4.7 months. In the cases which did not heal, only 1 complained of significant back pain at final follow up. The other 3 did not complain of pain at final follow up (Figures $3 \& 4$ ).

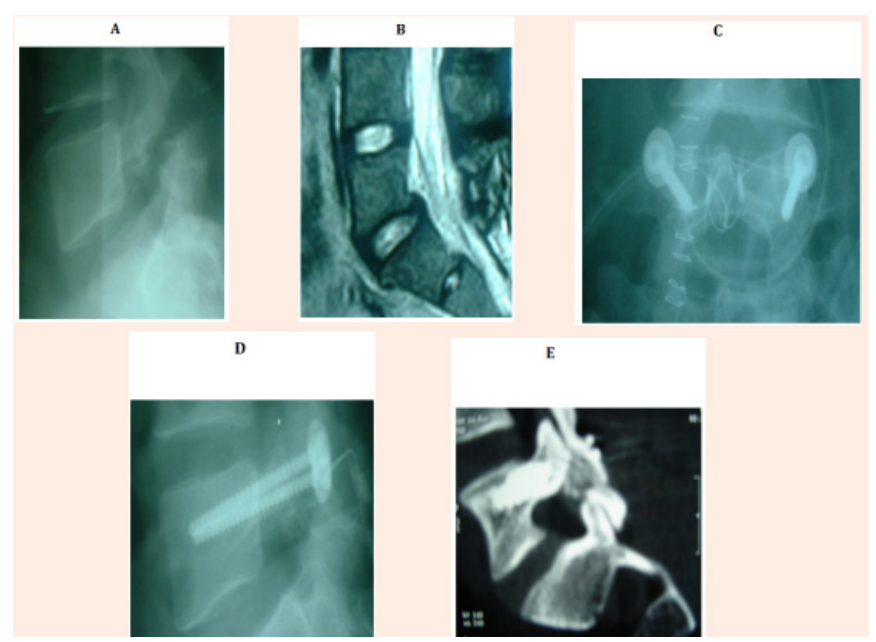

Figure 318 years old male with bilateral pars defect at L5 underwent the procedure. (A) Preoperative lateral plain radiograph showing the defect (B) Preoperative MRI shows intact intervening disc (C, D) Immediately postoperative plain radiographs $(\mathrm{E}) \mathrm{CT}$ I month later. Note the bone graft incorporating at the defect site.
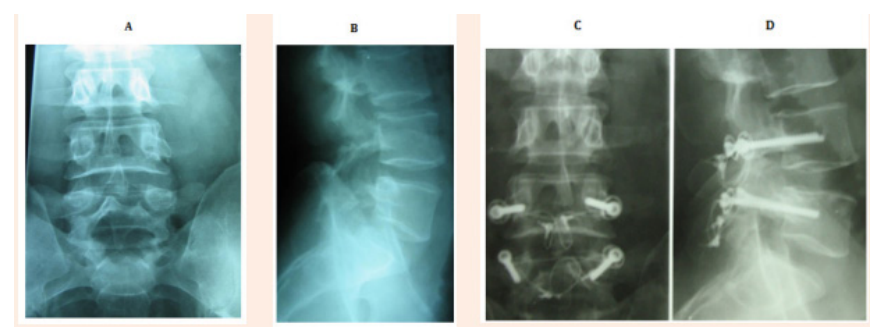

Figure 4 A 23 years old female with double level spodylolyis at L4 and L5. $(A, B)$ Preoperative plain radiographs. (C,D) 3 months after direct pars repair with cable screw construct at both levels.

\section{Discussion}

The concept of repairing pars defect is not new. Kimura et al. in 1968 described direct pars repair without instrumentation. ${ }^{15}$ The patients were confined to bed for 2 months and wore a corset for another 4 to 6 months. In 1970, Buck described bilateral screw fixation applied across the pars defect. ${ }^{9}$ The technique was surgically demanding while the screws interfered with the potential area for bone graft incorporation. Rajasekaran et al. ${ }^{16}$ performed this procedure in 9 patients. He reported excellent functional outcome in 5 and good outcome in 2. 
Scott technique involved passing wires around the transverse processes. ${ }^{10,11}$ Although excellent result had been reported, extensive dissection to completely expose the transverse process, proximity of the wire path to the nerve roots and the fixation depending on relatively weak bony elements of the spine (transverse and spinous processes) were problematic. Nozawa et al. ${ }^{17}$ used the segmental wiring fixation in 20 athletes. Bony union could be achieved in all cases with return to sport activities.

Morscher et al. ${ }^{12}$ described a technique using hook-screw construct. A modified Harrington hook was placed across the inferior edge of the lamina, and a screw was inserted into the superior articular process. Hefti et al.$^{18}$ reported $79 \%$ satisfactory relief of back pain and $73 \%$ radiological healing with better outcome in younger patients using this technique. However, there was $15 \%$ incidence of screws penetrating the inferior facet of the vertebra above.

In the face of technical difficulties with the previous methods, advances in spinal implants has allowed for emergence of techniques where the instruments provide strong anchors, are easily applicable, are of low profile and do not violate the facet joints. The constructs have to overcome the high stresses on the pars with extension and torsion. They should also not interfere with the surface area for bone grafting. ${ }^{14,19}$

Gillet et al. ${ }^{13}$ described a rod screws construct for pars repair. It involves insertion of pedicle screws into the affected vertebra and fixation of the loose posterior elements with a rod bent in $\mathrm{V}$ shape. They performed the procedure on 10 patients. Results were excellent in 6 patients with return to normal activities. The outcome was rated as good in one and fair in another. The procedure was considered a failure in a single patient although radiological healing could be achieved.

The cable screw construct also meets the above criteria. Excellent results have been reported with the use of this method. The technique has originally been reported by Songer et al. ${ }^{19}$ In a series of 7 patients, union could be achieved in all their cases. Clinically, 5 cases were rated as excellent and 2 as good according to the Prolo score. Bozarth et al $^{20}$ performed the procedure on three athletes with all three achieving radiological fusion, resolution of preoperative pain and return to sports. In our series radiological healing could be achieved in $86.6 \%$ of the cases with statistically significant improvement both in function and pain. Our study was conducted on larger series of patients as compared to other studies which stated $100 \%$ union rate. Furthermore, clinical improvement could even be noted in 3 of the patients who did not show union on images.

It is to be noted that the success of direct pars repair depends on proper patient selection besides the surgical technique. Poorer outcomes have been reported in patients with spondylolisthesis, degenerated discs and increasing age. ${ }^{10,11,18}$ In our series patients with degenerated discs and significant spondylolisthesis were excluded from the study. Furthermore, preoperative pars injection was performed in all cases as a predictor to success of the procedure. ${ }^{21}$

\section{Conclusion}

Favorable results have generally been reported with direct pars repair. However older techniques are surgically demanding with several limitations. The cable-screw construct appears to be a rigid, low profile construct with high success rates both radiologically and clinically.

\section{Acknowledgements}

None.

\section{Conflicts of interest}

None.

\section{References}

1. Wiltse LL, Rothman SLG. Spondylolisthesis: classification, diagnosis and natural history. Semin Spine Surg. 1989;1:78-94.

2. Rauch RA, Jinkins JR. Lumbosacral spondylolisthesis associated with spondylolysis. Neuroimaging Clin N Am. 1993;3:543-555.

3. Rossi F. Spondylolysis, spondylolisthesis and sports. J Sports Med Phys Fit. 1988;18:317-340.

4. Bradford DS. Management of spondylolysis and spondylolisthesis. Instr Course Lect. 1983;32:151-162.

5. Fredrickson BE, Baker D, McHolick WJ, et al. The natural history of spondylolysis and spondylolisthesis. J Bone Joint Surg Am. 1984;66(50:699-707.

6. Wang JM, Kim DJ, Yun YH. Posterior pedicle screw instrumentation and anterior interbody fusion in adult lumbar spondylolysis or grade 1 spondylolisthesis with segmental instability. J Spinal Disord. 1996;9:83-88.

7. Nachemson A. Repair of the spondylolisthetic defect and intertransversal fusion for young patients. ClinOrthop. 1976;117:101-105.

8. Mihara H, Onari K, Cheng BC, et al. The biomechanical effects of spondylolysis and its treatment. Spine. 2003;28:235-238.

9. Buck JE. Direct repair of the defect in spondylolisthesis. Preliminary report. J Bone Joint Surg. 1970;52(B):432-437.

10. Nicol RO, Scott JH. Lytic spondylolysis: Repair by wiring. Spine. 1986;11:1027-1030.

11. Johnson JV, Thompson AG. The Scott wiring technique for direct repair of lumbar spondylolysis.J Bone Joint Surg Br. 1992;74(3):426-430.

12. Morscher E, Gerber B, Fasel J. Surgical treatment of spondylolisthesis by bone grafting and direct stabilization by means of a hook screw. $A c-$ taOrthop Trauma Surg. 1984;103:175-178.

13. Gillet P, Petit M. Direct repair of spondylolysis without spondylolisthesis, using a rod-screw construct and bone grafting of the pars defect. Spine. 1999;24:1252-1256.

14. Deguchi M, Rapoff AJ, Zdeblick TA. Biomechanical comparison of spondylolysis fixation techniques. Spine. 1999;24:328-333.

15. Kimura M. My method of filling the lesion with spongy bone in spondylolysis and spondylolisthesis. Orthop Surg. 1968;19:285-295.

16. Rajaeskaran S, Subbiah M, Shetty AP. Direct repair of lumbar spondylolysis by Buck's technique. Indian J Orthop. 2011;45(2):136-140.

17. Nozawa S, Shimizu K, Miyamoto K, et al. Repair of pars interarticularis defect by segmental wire fixation in young athletes with spondylolysis. Am J Sports Med. 2003;31(3):359-364.

18. Hefti F, Seeling W, Morscher E. Repair of lumbar spondylolysis with a hook-screw. Int Orthop. 1992;16(1):81-85

19. Songer MN, Richard R. Repair of the pars interarticularis with a cablescrew construct: a preliminary report. Spine. 1998;23(2):263-269.

20. Bozarth GR, Fogel GR, Toohey JS, et al. Repair of pars interarticularis defect with a modified cable-screw construct.J SurgOrthop Adv. 2007;16(2):79-83.

21. Suh PB, Esses SI, Kostuik JP. Repair of pars interarticularis defect. The prognostic value of pars infiltration. Spine. 1991;16(8 suppl):445-448. 